\title{
Numerical Modelling of Influenza Model with Diffusion
}

\author{
Atit Jaichuang and Wirawan Chinviriyasit
}

\begin{abstract}
The SEIR model for the transmission dynamics of influenza is extended by the addition of second-order space derivatives to enable the geographic spread of the disease in a population which has not been vaccinated against it. The resulting system of four reaction-diffusion equations is solved by a convergent finite-difference technique which is first-order accurate in time and second-order accurate in space. The resulting methods are analyzed for local truncation errors and stability. The numerical results verify that the proposed method is more competitive in terms of numerical stability than the standard finite-difference method.
\end{abstract}

Index Terms-SEIR model, reaction-diffusion system, finite-difference method, initial condition, influenza, infectious diseases.

\section{INTRODUCTION}

Mathematical models for epidemics are tools that can be used to predict the epidemic outbreak which obtained from computer programming to calculate the various parameters such as the speed of the spread of the virus, density of population, age structure, distance and frequency of travel of the population in the area. Influenza has caused more morbidity and mortality than all other respiratory diseases. There are annual seasonal epidemics that cause about 500,000 deaths worldwide each year. The analysis of the spread of influenza epidemics dates back to the $18^{\text {th }}$ century. Since then different researchers developed these models on the basis of different type of anthropological parameters such as traffic patterns, contact patterns at work, in schools, at homes, at public places and the airline traffic (see, for instance, [1], [2]-[8]). These studies, however, gave no detail on the numerical method(s) used to solve the resulting nonlinear boundary-value problems (BVPS). This paper proposes an implicit finite difference method for solving the influenza epidemic model proposed in [9]. Although implicit by construction, the method can be implemented explicitly.

The model monitors four populations namely: the proportion of susceptible $S(x, t)$, exposed $E(x, t)$, infected $I(x, t)$ and recovered $R(x, t)$ individuals, respectively, at time $t$ and distance $x$ from the origin $(-L \leq x \leq L)$. The total population denoted $N(x, t)$ is given by $N(x, t)=S(x, t)+E(x, t)+I(x, t)+R(x, t)$. The influenza epidemic model (Massad et al. [10]) consists of the following equations, for all $t \geq 0$ and $-L \leq x \leq L$,

Manuscript received November 9, 2013; revised January 15, 2014

The authors are with the Department of Mathematics, King Mongkut's University of Technology Thonburi, Thung Khru, Bangkok 10140 Thailand (e-mail: atitjc@hotmail.com, iwirwong@kmutt.ac.th).

$$
\begin{gathered}
\frac{\partial S}{\partial t}=-\beta \frac{(E+I)}{N} S-\mu S+r N\left(1-\frac{N}{K}\right)+d_{1} \frac{\partial^{2} S}{\partial x^{2}}, \\
\frac{\partial E}{\partial t}=\beta \frac{(E+I)}{N} S-\omega_{1} E+d_{2} \frac{\partial^{2} E}{\partial x^{2}}, \\
\frac{\partial I}{\partial t}=\sigma E-\omega_{2} I+d_{3} \frac{\partial^{2} I}{\partial x^{2}}, \\
\frac{\partial R}{\partial t}=\kappa E+\gamma I-\mu R+d_{4} \frac{\partial^{2} R}{\partial x^{2}}
\end{gathered}
$$

where $\omega_{1}=(\mu+\sigma+\kappa), \omega_{2}=(\mu+\alpha+\gamma)$, and $d_{1}, \ldots, d_{4}$ are diffusion rates. For biological meaning, the parameters $\beta, \mu, r, K, \sigma, \kappa, \alpha$ and $\gamma$ denote infection rate, natural mortality rate, birth rate, carrying capacity, duration of latency, recovery rate of latent, flu induced mortality rate and recovery rate of clinically ill, respectively.

The initial conditions [11] are

$$
\begin{aligned}
& S(x, 0)=S_{0}, E(x, 0)=E_{0}, I(x, 0)=I_{0}, R(x, 0)=R_{0} ; \\
& -L \leq x \leq L,
\end{aligned}
$$

and the boundary conditions used for the domain are

$$
\begin{gathered}
\frac{\partial S(-L, t)}{\partial x}=\frac{\partial E(-L, t)}{\partial x}=\frac{\partial I(-L, t)}{\partial x}=\frac{\partial R(-L, t)}{\partial x}=0 ; t \geq 0, \\
\frac{\partial S(2, t)}{\partial x}=\frac{\partial E(2, t)}{\partial x}=\frac{\partial I(2, t)}{\partial x}=\frac{\partial R(2, t)}{\partial x}=0 ; t \geq 0
\end{gathered}
$$

\section{NUMERICAL METHODS}

\section{A. Discretization and Notations}

A solution of the system (1)-(6) may be computed by finite-difference methods by discretizing the space interval $[-L, L]$ into $M$ sub-intervals each of width $h>0$, and the time interval $t \geq 0$ is discretized in steps each of length $\ell>0$. The open region $\Omega=[-L, L] \times[t>0]$ and its boundary $\partial \Omega$ consisting of the lines $x=-L, x=L$ and $t=0$ are thus covered by a rectangular mesh, the mesh points having coordinates of the form $\left(x_{m}, t_{n}\right)=(m h, n \ell)$ where $\quad x_{m}=-L+m h(m=0,1,2, \ldots, M) \quad$ and $t_{n}=n \ell(n=0,1,2, \ldots)$. The solutions of (1)-(6) at the typical mesh point $\left(x_{m}, t_{n}\right)$ are, of course, $S\left(x_{m}, t_{n}\right), E\left(x_{m}, t_{n}\right), I\left(x_{m}, t_{n}\right)$ and $R\left(x_{m}, t_{n}\right)$ which will be denoted by $S_{m}^{n}, E_{m}^{n}, I_{m}^{n}$, and $R_{m}^{n}$, respectively. A family of numerical methods will be developed by approximating the time derivatives in (1)-(4) by the first order 
forward-difference replacement

$$
\frac{\partial u(x, t)}{\partial t}=\frac{u(x, t+\ell)-u(x, t)}{\ell}+O(\ell) \text { as } \ell \rightarrow 0,
$$

and the space derivatives in (1)-(4) by the weighted approximant

$$
\begin{aligned}
\frac{\partial^{2} u(x, t)}{\partial x^{2}} \approx & \frac{\theta\{u(x-h, t+\ell)-2 u(x, t+\ell)+u(x+h, t+\ell)\}}{h^{2}} \\
& +\frac{(1-\theta)\{u(x-h, t)-2 u(x, t)+u(x+h, t)\}}{h^{2}},
\end{aligned}
$$

in which $u(x, t)$ represents $S\left(x_{m}, t_{n}\right), E\left(x_{m}, t_{n}\right), I\left(x_{m}, t_{n}\right)$ or $R\left(x_{m}, t_{n}\right)$ and $0 \leq \theta \leq 1$ is a parameter. When $\theta=0,(8)$ is $O\left(h^{2}\right)$ as $h, \ell \rightarrow 0$ and is $O\left(h^{2}+\ell\right)$ as $h, \ell \rightarrow 0$ otherwise.

\section{B. Development of Finite-Difference Method}

A competitive finite-difference method for solving $S, E, I$, $R$ in (1)-(6), based on approximating the time derivative in (7) and the space derivative in (8) and making appropriate approximations for the right hand side, are given by

$$
\begin{aligned}
\frac{S_{m}^{n+1}-S_{m}^{n}}{\ell} & =-\beta \frac{\left(E_{m}^{n}+I_{m}^{n}\right)}{N_{m}^{n}} S_{m}^{n+1}-\mu S_{m}^{n+1} \\
& +r N_{m}^{n}\left(1-\frac{N_{m}^{n}}{K}\right)+d_{1} \theta\left(\frac{S_{m-1}^{n+1}-2 S_{m}^{n+1}+S_{m+1}^{n+1}}{h^{2}}\right)(9) \\
& +d_{1}(1-\theta)\left(\frac{S_{m-1}^{n}-2 S_{m}^{n}+S_{m+1}^{n}}{h^{2}}\right),
\end{aligned}
$$$$
\frac{E_{m}^{n+1}-E_{m}^{n}}{\ell}=\beta \frac{\left(E_{m}^{n+1}+I_{m}^{n}\right)}{N_{m}^{n}} S_{m}^{n}-(\mu+\sigma+\kappa) E_{m}^{n+1}
$$$$
+d_{2} \theta\left(\frac{E_{m-1}^{n+1}-2 E_{m}^{n+1}+E_{m+1}^{n+1}}{h^{2}}\right)
$$$$
+d_{2}(1-\theta)\left(\frac{E_{m-1}^{n}-2 E_{m}^{n}+E_{m+1}^{n}}{h^{2}}\right) \text {, }
$$

$$
\begin{aligned}
\frac{I_{m}^{n+1}-I_{m}^{n}}{\ell}= & \sigma E_{m}^{n}-(\mu+\alpha+\gamma) I_{m}^{n+1} \\
& +d_{3} \theta\left(\frac{I_{m-1}^{n+1}-2 I_{m}^{n+1}+I_{m+1}^{n+1}}{h^{2}}\right) \\
& +d_{3}(1-\theta)\left(\frac{I_{m-1}^{n}-2 I_{m}^{n}+I_{m+1}^{n}}{h^{2}}\right),
\end{aligned}
$$$$
\frac{R_{m}^{n+1}-R_{m}^{n}}{\ell}=\kappa E_{m}^{n}+\gamma I_{m}^{n}-\mu R_{m}^{n+1}
$$$$
+d_{4} \theta\left(\frac{R_{m-1}^{n+1}-2 R_{m}^{n+1}+R_{m+1}^{n+1}}{h^{2}}\right)
$$$$
+d_{4}(1-\theta)\left(\frac{R_{m-1}^{n}-2 R_{m}^{n}+R_{m+1}^{n}}{h^{2}}\right) \text {, }
$$

Equations (9)-(12) may be re-arranged to give, for $p=\ell / h^{2}, m=0,1,2, \ldots, M, n=1,2, \ldots$,

$$
\begin{gathered}
-d_{1} p \theta S_{m-1}^{n+1}+d_{S} S_{m}^{n+1}-d_{1} p \theta S_{m+1}^{n+1}=d_{1} p(1-\theta) S_{m-1}^{n} \\
+\left[1-2 d_{1} p(1-\theta)\right] S_{m}^{n}+d_{1}(1-\theta) S_{m+1}^{n}+\ell r A_{m}^{n} \\
-d_{2} p \theta E_{m-1}^{n+1}+d_{E} E_{m}^{n+1}-d_{2} p \theta E_{m+1}^{n+1}=d_{2} p(1-\theta) E_{m-1}^{n} \\
+\left[1-2 d_{2} p(1-\theta)\right] E_{m}^{n}+d_{2}(1-\theta) E_{m+1}^{n}+\ell \beta I_{m}^{n} C_{m}^{n} \\
-d_{3} p \theta I_{m-1}^{n+1}+d_{I} I_{m}^{n+1}-d_{3} p \theta I_{m+1}^{n+1}=d_{3} p(1-\theta) I_{m-1}^{n} \\
+\left[1-2 d_{3} p(1-\theta)\right] I_{m}^{n}+d_{3}(1-\theta) I_{m+1}^{n}+\ell \sigma E_{m}^{n}, \\
-d_{4} p \theta R_{m-1}^{n+1}+d_{R} R_{m}^{n+1}-d_{4} p \theta R_{m+1}^{n+1}=d_{4} p(1-\theta) R_{m-1}^{n} \\
+\left[1-2 d_{4} p(1-\theta)\right] R_{m}^{n}+d_{4}(1-\theta) R_{m+1}^{n}+\ell \kappa E_{m}^{n}+\ell \gamma I_{m}^{n},
\end{gathered}
$$

where $A_{m}^{n}=N_{m}^{n}\left(1-\frac{N_{m}^{n}}{K}\right), B_{m}^{n}=\frac{E_{m}^{n}+I_{m}^{n}}{N_{m}^{n}}, C_{m}^{n}=\frac{S_{m}^{n}}{N_{m}^{n}}$,

$$
\begin{aligned}
& d_{S}=1+2 d_{1} p \theta+\ell \mu+\ell \beta B_{m}^{n}, d_{E}=1+2 d_{2} p \theta+\ell \omega_{1}-\ell \beta C_{m}^{n}, \\
& d_{I}=1+2 d_{3} p \theta+\ell \omega_{2}, d_{R}=1+2 d_{4} p \theta+\ell \mu .
\end{aligned}
$$

The method (13)-(16) is denoted as method $D M(\theta)$. Verification of accuracy may be obtained by considering the local truncation errors,

$$
\begin{gathered}
\mathscr{L}_{S}=\mathscr{L}_{S}[S(x, t), E(x, t), I(x, t), R(x, t) ; h, \ell], \\
\mathscr{L}_{E}=\mathscr{L}_{E}[S(x, t), E(x, t), I(x, t), R(x, t) ; h, \ell], \\
\mathscr{L}_{I}=\mathscr{L}_{I}[S(x, t), E(x, t), I(x, t), R(x, t) ; h, \ell], \\
\text { and } \mathscr{L}_{R}=\mathscr{L}_{R}[S(x, t), E(x, t), I(x, t), R(x, t) ; h, \ell],
\end{gathered}
$$

associated with (13)-(16) at the point $(x, t)=\left(x_{m}, t_{n}\right)$, respectively, which may be obtained from (9) -(12). Using Taylor series expansion in (9)-(12) lead to

$$
\begin{gathered}
\mathscr{L}_{S}=\frac{-1}{12} d_{1} h^{2} \frac{\partial^{4} S}{\partial x^{4}} \\
+\ell\left[\frac{1}{2} \frac{\partial^{2} S}{\partial t^{2}}+\left(\beta \frac{(E+I)}{N}+\mu\right) \frac{\partial S}{\partial t}-d_{1} \theta \frac{\partial^{3} S}{\partial x^{2} \partial t}\right]+\cdots \\
\mathscr{L}_{E}=\frac{-1}{12} d_{2} h^{2} \frac{\partial^{4} E}{\partial x^{4}} \\
+\ell\left[\frac{1}{2} \frac{\partial^{2} E}{\partial t^{2}}+\omega_{1} \frac{\partial E}{\partial t}-d_{2} \theta \frac{\partial^{3} E}{\partial x^{2} \partial t}\right]+\cdots \\
\mathscr{L}_{I}=\frac{-1}{12} d_{3} h^{2} \frac{\partial^{4} I}{\partial x^{4}}+\ell\left[\frac{1}{2} \frac{\partial^{2} I}{\partial t^{2}}+\omega_{2} \frac{\partial I}{\partial t}-d_{3} \theta \frac{\partial^{3} I}{\partial x^{2} \partial t}\right]+\cdots, \\
\mathscr{L}_{R}=\frac{-1}{12} d_{4} h^{2} \frac{\partial^{4} R}{\partial x^{4}}+\ell\left[\frac{1}{2} \frac{\partial^{2} R}{\partial t^{2}}-\mu \frac{\partial R}{\partial t}-d_{4} \theta \frac{\partial^{3} R}{\partial x^{2} \partial t}\right]+\cdots,
\end{gathered}
$$

Equations (17)-(20) verify that method $D M(\theta)$ is $O\left(h^{2}+\ell\right)$

as $h, \ell \rightarrow 0$.

\section{Standard Finite-Difference Method}

The corresponding standard finite-difference method for solving the initial/boundary value problems (IBVP) obtained 
by using (7) and (8) in (1)-(4) and evaluating the right-hand functions in (1)-(4) at base time level $t=t_{n}$, are given by, respectively, for $\quad p=\ell / h^{2}, m=0,1,2, \ldots, M \quad$ and $n=0,1,2, \ldots$

$$
\begin{gathered}
-d_{1} p \theta S_{m-1}^{n+1}+\left(1+2 d_{1} p \theta\right) S_{m}^{n+1}-d_{1} p \theta S_{m+1}^{n+1}=d_{1} p(1-\theta) S_{m-1}^{n} \\
+\left(d_{S S}-\ell \beta B_{m}^{n}\right) S_{m}^{n}+d_{1}(1-\theta) S_{m+1}^{n}+\ell r A_{m}^{n}, \\
-d_{2} p \theta E_{m-1}^{n+1}+\left(1+2 d_{2} p \theta\right) E_{m}^{n+1}-d_{2} p \theta E_{m+1}^{n+1}=d_{2} p(1-\theta) E_{m-1}^{n} \\
+\left(d_{E E}+\ell \beta C_{m}^{n}\right) E_{m}^{n}+d_{2}(1-\theta) E_{m+1}^{n}+\ell \beta I_{m}^{n} C_{m}^{n}, \quad(22) \\
-d_{3} p \theta I_{m-1}^{n+1}+\left(1+2 d_{3} p \theta\right) I_{m}^{n+1}-d_{3} p \theta I_{m+1}^{n+1}=d_{3} p(1-\theta) I_{m-1}^{n} \\
+d_{E E} I_{m}^{n}+d_{3}(1-\theta) I_{m+1}^{n}+\ell \sigma E_{m}^{n}, \\
-d_{4} p \theta R_{m-1}^{n+1}+\left(1+2 d_{4} p \theta\right) R_{m}^{n+1}-d_{4} p \theta R_{m+1}^{n+1}=d_{4} p(1-\theta) R_{m-1}^{n} \\
+d_{R R} R_{m}^{n}+d_{4}(1-\theta) R_{m+1}^{n}+\ell \kappa E_{m}^{n}+\ell \gamma I_{m}^{n},
\end{gathered}
$$

where

$$
\begin{aligned}
d_{S S}= & 1-\ell \mu-2 d_{1} p(1-\theta), d_{E E}=1-\ell \omega_{1}-2 d_{2} p(1-\theta), \\
& d_{I I}=1-\ell \omega_{2}-2 d_{3} p(1-\theta), d_{R R}=1-\ell \mu-2 d_{4} p(1-\theta),
\end{aligned}
$$

The method (21)-(24) is denoted as method $S M(\theta)$.

Using Taylor series expansion, the associated local truncation errors at the point $(x, t)=\left(x_{m}, t_{n}\right)$ of the method $\operatorname{SM}(\theta)$ are, respective,

$$
\begin{aligned}
& \mathscr{L}_{S}=\frac{-1}{12} d_{1} h^{2} \frac{\partial^{4} S}{\partial x^{4}}+\ell\left[\frac{1}{2} \frac{\partial^{2} S}{\partial t^{2}}-d_{1} \theta \frac{\partial^{3} S}{\partial x^{2} \partial t}\right]+\cdots \\
& \mathscr{L}_{E}=\frac{-1}{12} d_{2} h^{2} \frac{\partial^{4} E}{\partial x^{4}}+\ell\left[\frac{1}{2} \frac{\partial^{2} E}{\partial t^{2}}-d_{2} \theta \frac{\partial^{3} E}{\partial x^{2} \partial t}\right]+\cdots \\
& \mathscr{L}_{I}=\frac{-1}{12} d_{3} h^{2} \frac{\partial^{4} I}{\partial x^{4}}+\ell\left[\frac{1}{2} \frac{\partial^{2} I}{\partial t^{2}}-d_{3} \theta \frac{\partial^{3} I}{\partial x^{2} \partial t}\right]+\cdots \\
& \mathscr{L}_{R}=\frac{-1}{12} d_{4} h^{2} \frac{\partial^{4} R}{\partial x^{4}}+\ell\left[\frac{1}{2} \frac{\partial^{2} R}{\partial t^{2}}-d_{4} \theta \frac{\partial^{3} R}{\partial x^{2} \partial t}\right]+\cdots
\end{aligned}
$$

Equations (25)-(28) verify that method $S M(\theta)$ is $O\left(h^{2}+\ell\right)$ as $h, \ell \rightarrow 0$.

\section{Stability Analyses}

The von Neumann or Fourier series method seek the condition(s) under which small errors of the forms,

$$
\begin{gathered}
Z_{S, m}^{n}:=S_{m}^{n}-\tilde{S}_{m}^{n}=e^{\psi_{1} n \ell} e^{i \delta_{1} m h}, \\
Z_{E, m}^{n}:=E_{m}^{n}-\tilde{E}_{m}^{n}=e^{\psi_{2} n \ell} e^{i \delta_{2} m h}, \\
Z_{I, m}^{n}:=I_{m}^{n}-\tilde{I}_{m}^{n}=e^{\psi_{3} n \ell} e^{i \delta_{3} m h}, \\
Z_{R, m}^{n}:=R_{m}^{n}-\tilde{R}_{m}^{n}=e^{\psi_{4} n \ell} e^{i \delta_{4} m h},
\end{gathered}
$$

where

$$
\psi_{1}, \psi_{2}, \psi_{3}, \psi_{4}, \delta_{1}, \delta_{2}, \delta_{3} \text { and } \delta_{4} \text { are real, } i=\sqrt{-1} \text { and }
$$

$\tilde{S}_{m}, \tilde{E}_{m}, \tilde{I}_{m}, \tilde{R}_{m}$ are a perturbed numerical solutions. The necessary conditions for the errors not to grow as $n \rightarrow \infty$ are

$$
\begin{aligned}
& \left|e^{\psi_{1} \ell}\right| \leq 1+M_{S} \ell,\left|e^{\psi_{2} \ell}\right| \leq 1+M_{E} \ell,\left|e^{\psi_{3} \ell}\right| \leq 1+M_{I} \ell, \\
& \left|e^{\psi_{4} \ell}\right| \leq 1+M_{R} \ell
\end{aligned}
$$

where $M_{S}, M_{E}, M_{I}, M_{R}$ are non-negative constants independent of $h$ and $\ell$. The conditions in (33) make no allowance for growing solutions if $M_{S}=0, M_{E}=0, M_{I}=0$ and $M_{R}=0$.

Method: $D M(\theta)$ : Substituting $Z_{S}$ into (21) leads to the (local) stability equation

$$
\begin{aligned}
\left\{1+\ell \mu+\ell \beta B_{m}^{n}+\right. & \left.4 d_{1} p \theta \sin ^{2} \frac{\delta_{1} h}{2}\right\} \zeta_{S} \\
& =1-4 d_{1} p(1-\theta) \sin ^{2} \frac{\delta_{1} h}{2}
\end{aligned}
$$

where $\xi_{S}=e^{\psi_{1} \ell}$ and $B_{m}^{n}$ are treated as a (local) constant. The von Neumann necessary condition for stability is $\left|\xi_{S}\right|<1$, that is, the stability restrictions are

$$
\begin{array}{r}
0 \leq \theta<1 / 2, \quad p \leq \frac{2+\ell \mu+\ell \beta B_{m}^{n}}{4 d_{1}(1-2 \theta)}, \\
\theta=1 / 2, \quad-\ell \mu-\ell \beta B_{m}^{n} \leq 2, \quad p>0, \\
1 / 2<\theta \leq 1, \quad p \geq \frac{-\ell \mu-\ell \beta B_{m}^{n}-2}{4 d_{1}(2 \theta-1)} .
\end{array}
$$

Substituting $Z_{E}$ into (22) gives the (local) stability equation

$$
\begin{aligned}
\left\{1+\ell \omega_{1}+4 d_{2} p \theta \sin ^{2} \frac{\delta_{2} h}{2}\right\} \zeta_{E} & =1+\ell \beta C_{m}^{n} \\
& -4 d_{2} p(1-\theta) \sin ^{2} \frac{\delta_{2} h}{2},
\end{aligned}
$$

where $\xi_{E}=e^{\psi_{2} \ell}$ and $C_{m}^{n}$ is treated as a (local) constant. The von Neumann necessary condition for stability is $\left|\xi_{E}\right|<1$, that is, the stability restrictions are

$$
0 \leq \theta<1 / 2, \quad p \leq \frac{2+\ell \omega_{1}+\ell \beta C_{m}^{n}}{4 d_{2}(1-2 \theta)} \text { and } p \geq \frac{-\ell \omega_{1}+\ell \beta C_{m}^{n}}{4 d_{2}},
$$

$\theta=1 / 2, \quad 2+\ell \omega_{1}+\ell \beta C_{m}^{n} \geq 0$ and $p \geq \frac{-\ell \omega_{1}+\ell \beta C_{m}^{n}}{4 d_{2}}$

$1 / 2<\theta \leq 1, \quad p \leq \frac{-2-\ell \omega_{1}-\ell \beta C_{m}^{n}}{4 d_{2}(2 \theta-1)}$ and $p \geq \frac{-\ell \omega_{1}+\ell \beta C_{m}^{n}}{4 d_{2}}$.

Substituting $Z_{I}$ into (23) gives the (local) stability equation

$\left\{1+\ell \omega_{2}+4 d_{3} p \theta \sin ^{2} \frac{\delta_{3} h}{2}\right\} \zeta_{I}=1-4 d_{3} p(1-\theta) \sin ^{2} \frac{\delta_{3} h}{2}$, 
where $\xi_{I}=e^{\psi_{3} \ell}$. The von Neumann necessary condition for stability is $\left|\xi_{I}\right|<1$, that is, the stability restrictions are

$$
\begin{aligned}
0 \leq \theta<1 / 2, & p \leq \frac{2+\ell \omega_{2}}{4 d_{3}(1-2 \theta)}, \\
\theta=1 / 2, & 2+\ell \omega_{2} \geq 0, p>0, \\
1 / 2<\theta \leq 1, & p \leq \frac{-2-\ell \omega_{2}}{4 d_{3}(2 \theta-1)} .
\end{aligned}
$$

Substituting $Z_{R}$ into (24) gives the (local) stability equation

$\left\{1+\ell \mu+4 d_{4} p \theta \sin ^{2} \frac{\delta_{4} h}{2}\right\} \zeta_{R}=1-4 d_{4} p(1-\theta) \sin ^{2} \frac{\delta_{4} h}{2}$,

where $\xi_{R}=e^{\psi_{4} \ell}$. The von Neumann necessary condition for stability is $\left|\xi_{R}\right|<1$, that is, the stability restrictions are

$$
\begin{aligned}
0 \leq \theta<1 / 2, & p \leq \frac{2-\ell \mu}{4 d_{4}(1-2 \theta)}, \\
\theta=1 / 2, & 2-\ell \mu \geq 0, p>0, \\
1 / 2<\theta \leq 1, & p \leq \frac{\ell \mu-2}{4 d_{4}(2 \theta-1)} .
\end{aligned}
$$

Method: $\operatorname{SM}(\theta)$ : Similarly, substituting $Z_{S}, Z_{E}, Z_{I}, Z_{R}$ into (21),(22),(23) and (24), respectively, yield the von Neumann stability restrictions as follows.

The stability restrictions of the method for $S$ in (13) are

$$
\begin{aligned}
& 0 \leq \theta<1 / 2, \quad p \leq \frac{2-\ell \mu-\ell \beta B_{m}^{n}}{4 d_{1}(1-2 \theta)}, \\
& \theta=1 / 2, \quad \ell \mu+\ell \beta B_{m}^{n} \leq 2, \quad p>0, \\
& 1 / 2<\theta \leq 1, \quad p \geq \frac{\ell \mu+\ell \beta B_{m}^{n}+2}{4 d_{1}(2 \theta-1)} .
\end{aligned}
$$

The stability restrictions of the method for $E$ in (14) are $0 \leq \theta<1 / 2, p \leq \frac{2-\ell \omega_{1}+\ell \beta C_{m}^{n}}{4 d_{2}(1-2 \theta)}$ and $p \geq \frac{-\ell \omega_{1}+\ell \beta C_{m}^{n}}{4 d_{2}}$,

$$
\theta=1 / 2, \quad 2-\ell \omega_{1}+\ell \beta C_{m}^{n} \geq 0 \text { and } p \geq \frac{-\ell \omega_{1}+\ell \beta C_{m}^{n}}{4 d_{2}},
$$

$1 / 2<\theta \leq 1, \quad p \leq \frac{-2+\ell \omega_{1}-\ell \beta C_{m}^{n}}{4 d_{2}(2 \theta-1)}$ and $p \geq \frac{-\ell \omega_{1}+\ell \beta C_{m}^{n}}{4 d_{2}}$.

The stability restrictions of the method for $I(15)$ are

$$
\begin{aligned}
& 0 \leq \theta<1 / 2, \quad p \leq \frac{2-\ell \omega_{2}}{4 d_{3}(1-2 \theta)}, \\
& \theta=1 / 2, \quad 2-\ell \omega_{2} \geq 0, \quad p>0, \\
& 1 / 2<\theta \leq 1, \quad p \leq \frac{-2+\ell \omega_{2}}{4 d_{3}(2 \theta-1)} .
\end{aligned}
$$

The stability restrictions of the method for $R(16)$ are

$$
\begin{aligned}
& 0 \leq \theta<1 / 2, \quad p \leq \frac{2-\ell \mu}{4 d_{4}(1-2 \theta)}, \\
& \theta=1 / 2, \quad 2-\ell \mu \geq 0, \quad p>0 \\
& 1 / 2<\theta \leq 1, \quad p \leq \frac{-2+\ell \mu}{4 d_{4}(2 \theta-1)}
\end{aligned}
$$

It is followed that the stability intervals of the method $\operatorname{SM}(\theta)$ and $\operatorname{DM}(\theta)$ are given by the intersection of the restriction on $p$ in each value of $h$, see Equations (42), (43), (44) and (45) and (35), (37), (39) and (41), respectively. These result show that the method $\operatorname{DM}(\theta)$ is seen to be more competitive (in terms of stability intervals) than the method $\operatorname{SM}(\theta)$. Numerical simulations is demonstrated in Section III to confirm these results.

\section{IMPLEMENTATION}

The derivative $\frac{\partial S}{\partial x}$ in (6)-(7) may be approximated by the second-order, central-difference replacement

$$
\frac{\partial S(x, t)}{\partial x}=\frac{S(x+h, t)-S(x-h, t)}{2 h}+O\left(h^{2}\right),
$$

with a similar replacement being made to $\frac{\partial E}{\partial x}, \frac{\partial I}{\partial x}$ and $\frac{\partial R}{\partial x}$. The implementation of the boundary conditions (6)-(7) to second-order, yield, on $x=-L$ and $x=L$, for $n=0,1,2, \ldots$

$$
\begin{aligned}
& S_{1}^{n}=S_{-1}^{n}, E_{1}^{n}=E_{-1}^{n}, I_{1}^{n}=I_{-1}^{n}, R_{1}^{n}=R_{-1}^{n} \text { and } \\
& S_{M+1}^{n}=S_{M-1}^{n}, E_{M+1}^{n}=E_{M-1}^{n}, I_{M+1}^{n}=I_{M-1}^{n}, R_{M+1}^{n}=R_{M-1}^{n} .
\end{aligned}
$$

Thus, equation (47) introduce the exterior grid points $\left(x_{-1}, t\right)=(-h, n \ell)$ and $\left(x_{M+1}, t\right)=((M+1) h, n \ell)$. let

$$
\begin{aligned}
& \mathbf{S}^{n+1}=\left[S_{0}^{n+1}, S_{1}^{n+1}, \ldots, S_{M}^{n+1}\right]^{T}, \mathbf{E}^{n+1}=\left[E_{0}^{n+1}, E_{1}^{n+1}, \ldots, E_{M}^{n+1}\right]^{T}, \\
& \mathbf{I}^{n+1}=\left[I_{0}^{n+1}, I_{1}^{n+1}, \ldots, I_{M}^{n+1}\right]^{T} \text { and } \mathbf{R}^{n+1}=\left[R_{0}^{n+1}, R_{1}^{n+1}, \ldots, R_{M}^{n+1}\right]^{T}
\end{aligned}
$$

where $T$ denotes transpose. The implementation of methods $\operatorname{SM}(\theta)$ and $\operatorname{DM}(\theta)$, are as follows.

Method: $\operatorname{SM}(\theta)$ :

Taking $m=0, M$ in (21)-(24) and using (47) gives $m=0$,

$$
\begin{aligned}
&\left(1+2 d_{1} p \theta\right) S_{0}^{n+1}-2 d_{1} p \theta S_{1}^{n+1}=\left(d_{S S S}-\ell \beta B_{0}^{n}\right) S_{0}^{n} \\
&+ 2 d_{1}(1-\theta) S_{1}^{n}+\ell r A_{0}^{n}, \\
&\left(1+2 d_{2} p \theta\right) E_{0}^{n+1}-2 d_{2} p \theta E_{1}^{n+1}=\left(d_{E E E}+\ell \beta C_{0}^{n}\right) E_{0}^{n} \\
&+2 d_{2}(1-\theta) E_{1}^{n}+\ell \beta I_{0}^{n} C_{0}^{n}, \\
&\left(1+2 d_{3} p \theta\right) I_{0}^{n+1}-2 d_{3} p \theta I_{1}^{n+1}= d_{I I I} I_{0}^{n}+2 d_{3} p(1-\theta) I_{1}^{n} \\
&+\ell \sigma E_{0}^{n}, \\
& \\
&\left(1+2 d_{4} p \theta\right) R_{0}^{n+1}-2 d_{4} p \theta R_{1}^{n+1}= d_{R R R} R_{0}^{n}+2 d_{4}(1-\theta) R_{1}^{n} \\
&+\ell \kappa E_{0}^{n}+\ell \gamma I_{0}^{n},
\end{aligned}
$$

and $m=M$,

$$
\begin{array}{r}
-2 d_{1} p \theta S_{M-1}^{n+1}+\left(1+2 d_{1} p \theta\right) S_{M}^{n+1}=2 d_{1}(1-\theta) S_{M-1}^{n} \\
+\left(d_{S S S}-\ell \beta B_{M}^{n}\right) S_{M}^{n},+\ell r A_{M}^{n},
\end{array}
$$




$$
\begin{array}{r}
-2 d_{2} p \theta E_{M-1}^{n+1}+\left(1+2 d_{2} p \theta\right) E_{M}^{n+1}=2 d_{2}(1-\theta) E_{M-1}^{n} \\
+\left(d_{E E E}+\ell \beta C_{M}^{n}\right) E_{M}^{n}+\ell \beta I_{M}^{n} C_{M}^{n}, \\
-2 d_{3} p \theta I_{M-1}^{n+1}+\left(1+2 d_{3} p \theta\right) I_{M}^{n+1}=2 d_{3} p(1-\theta) I_{M-1}^{n} \\
+d_{I I I I} I_{M}^{n}+\ell \sigma E_{M}^{n}, \\
-2 d_{4} p \theta R_{M-1}^{n+1}+\left(1+2 d_{4} p \theta\right) R_{M}^{n+1}=2 d_{4}(1-\theta) R_{M-1}^{n} \\
+d_{R R R} R_{M}^{n}+\ell \kappa E_{M}^{n}+\ell \gamma I_{M}^{n},
\end{array}
$$

where

$$
\begin{aligned}
d_{S S S}= & 1-\ell \mu-2 d_{1} p(1-\theta), d_{E E E}=1-\ell \omega_{1}-2 d_{2} p(1-\theta), \\
& d_{I I I}=1-\ell \omega_{2}-2 d_{3} p(1-\theta), d_{R R R}=1-\ell \mu-2 d_{4} p(1-\theta),
\end{aligned}
$$

Thus, from (21)-(24) for and $m=1, \ldots, M-1$, and (48) -(51)the solution vectors $\mathbf{S}^{n+1}, \mathbf{E}^{n+1}, \mathbf{I}^{n+1}$ and $\mathbf{R}^{n+1}$ may be obtained using the following parallel algorithm:

Processor 1: Solve $A_{1} \mathbf{S}^{n+1}=B_{1} \mathbf{S}^{n}+\mathbf{q}_{1}$ for $\mathbf{S}^{n+1}$,

Processor 2: Solve $A_{2} \mathbf{E}^{n+1}=B_{2} \mathbf{E}^{n}+\mathbf{q}_{2}$ for $\mathbf{E}^{n+1}$,

Processor 3: Solve $A_{3} \mathbf{I}^{n+1}=B_{3} \mathbf{I}^{n}+\mathbf{q}_{3}$ for $\mathbf{I}^{n+1}$,

Processor 4: Solve $A_{4} \mathbf{R}^{n+1}=B_{4} \mathbf{R}^{n}+\mathbf{q}_{4}$ for $\mathbf{R}^{n+1}$,

where $A_{1}, A_{2}, A_{3}, A_{4}$ are a constant, tridiagonal matrix of order $M+1$ and

$$
\begin{aligned}
& \mathbf{q}_{\mathbf{1}}=\left[\ell r A_{0}^{n}, \ldots, \ell r A_{M}^{n}\right]^{T}, \\
& \mathbf{q}_{\mathbf{2}}=\left[\ell \beta I_{0}^{n} C_{0}^{n}, \ldots, \ell \beta I_{M}^{n} C_{M}^{n}\right]^{T}, \\
& \mathbf{q}_{\mathbf{3}}=\left[\ell \sigma E_{0}^{n}, \ldots, \ell \sigma E_{M}^{n}\right]^{T}, \\
& \mathbf{q}_{\mathbf{4}}=\left[\ell \kappa E_{0}^{n}+\ell \gamma I_{0}^{n}, \ldots, \ell \kappa E_{M}^{n}+\ell \gamma I_{M}^{n}\right]^{T},
\end{aligned}
$$

$T$ denoting transpose, is a constant vector of order $M+1$. The square matrices $B_{1}=B_{1}\left(\mathbf{S}^{n}\right), B_{2}=B_{2}\left(\mathbf{E}^{n}\right)$,

$B_{3}=B_{3}\left(\mathbf{I}^{n}\right)$ and $B_{4}=B_{4}\left(\mathbf{R}^{n}\right)$ are also of order $M+1$.

The element of $A_{i}, B_{i}, i=1, \ldots 4$, are easily obtained from (47)-(54) and (21)-(24) with $m=1,2, \ldots, M$.

Method: $D M(\theta)$ :

Taking $m=0, M$ in (13)-(16) and using (47) gives $m=0$,

$$
\begin{aligned}
\left(d_{S}+\ell \beta B_{0}^{n}\right) S_{0}^{n+1}-2 d_{1} p \theta S_{1}^{n+1} & =\left\{1-2 d_{1} p(1-\theta)\right\} S_{0}^{n} \\
& +2 d_{1}(1-\theta) S_{1}^{n}+\ell r A_{0}^{n}, \\
\left(d_{E}-\ell \beta C_{0}^{n}\right) E_{0}^{n+1}-2 d_{2} p \theta E_{1}^{n+1} & =\left\{1-2 d_{2} p(1-\theta)\right\} E_{m}^{n} \\
+ & 2 d_{2}(1-\theta) E_{1}^{n}+\ell \beta I_{0}^{n} C_{0}^{n}, \\
d_{I} I_{0}^{n+1}-2 d_{3} p \theta I_{1}^{n+1} & =\left\{1-2 d_{3} p(1-\theta)\right\} I_{0}^{n} \\
& +2 d_{3} p(1-\theta) I_{1}^{n}+\ell \sigma E_{0}^{n},
\end{aligned}
$$

$$
\begin{aligned}
d_{R} R_{0}^{n+1}-2 d_{4} p & \theta R_{1}^{n+1}=\left\{1-2 d_{4} p(1-\theta)\right\} R_{0}^{n} \\
+ & 2 d_{4}(1-\theta) R_{1}^{n}+\ell \kappa E_{0}^{n}+\ell \gamma I_{0}^{n},
\end{aligned}
$$

and $m=M$,

$$
\begin{array}{r}
-2 d_{1} p \theta S_{M-1}^{n+1}+\left(d_{S}+\ell \beta B_{M}^{n}\right) S_{M}^{n+1}=2 d_{1}(1-\theta) S_{M-1}^{n} \\
+\left\{1-2 d_{1} p(1-\theta)\right\} S_{M}^{n}+\ell r A_{M}^{n}, \\
-2 d_{2} p \theta E_{M-1}^{n+1}+\left(d_{E}-\ell \beta C_{M}^{n}\right) E_{M}^{n+1}=2 d_{2}(1-\theta) E_{M-1}^{n} \\
+\left\{1-2 d_{2} p(1-\theta)\right\} E_{M}^{n}+\ell \beta I_{M}^{n} C_{M}^{n}, \\
-2 d_{3} p \theta I_{M-1}^{n+1}+d_{I} I_{M}^{n+1}=2 d_{3} p(1-\theta) I_{M-1}^{n} \\
+\left\{1-2 d_{3} p(1-\theta)\right\} I_{M}^{n}+\ell \sigma E_{M}^{n}, \\
-2 d_{4} p \theta R_{M-1}^{n+1}+d_{R} R_{M}^{n+1}=2 d_{4}(1-\theta) R_{M-1}^{n} \\
+\left\{1-2 d_{4} p(1-\theta)\right\} R_{M}^{n}+\ell \kappa E_{M}^{n}+\ell \gamma I_{M}^{n},
\end{array}
$$

In this method the solution vectors $\mathbf{S}^{n+1}, \mathbf{E}^{n+1}, \mathbf{I}^{n+1}$ and $\mathbf{R}^{n+1}$ may be obtained using the parallel algorithm:

Processor 1: Solve $C_{1} \mathbf{S}^{n+1}=D_{1} \mathbf{S}^{n}+\mathbf{q}_{1}$ for $\mathbf{S}^{n+1}$,

Processor 2: Solve $C_{2} \mathbf{E}^{n+1}=D_{2} \mathbf{E}^{n}+\mathbf{q}_{2}$ for $\mathbf{E}^{n+1}$,

Processor 3: Solve $C_{3} \mathbf{I}^{n+1}=D_{3} \mathbf{I}^{n}+\mathbf{q}_{3}$ for $\mathbf{I}^{n+1}$,

Processor 4: Solve $C_{4} \mathbf{R}^{n+1}=D_{4} \mathbf{R}^{n}+\mathbf{q}_{4}$ for $\mathbf{R}^{n+1}$,

in which the matrices $C_{i}, D_{i}, i=1, \ldots 4$, are square and of order $M+1$. The element of $D_{1}=D_{1}\left(\mathbf{S}^{n}\right), D_{2}=D_{2}\left(\mathbf{E}^{n}\right)$,

$D_{3}=D_{3}\left(\mathbf{I}^{n}\right)$ and $D_{4}=D_{4}\left(\mathbf{R}^{n}\right)$, and the constant matrices $C_{i}, i=1, \ldots 4$, may be obtained from (61)-(68), (13)-(16) with $m=1,2, \ldots, M$.

As described above, the linear algebraic systems given by (56)-(59) and (69)-(72) can be solved using parallel computation (using a computer with two processors). In parallel computation, the vectors $\mathbf{S}^{n+1}, \mathbf{E}^{n+1}, \mathbf{I}^{n+1}$ and $\mathbf{R}^{n+1}$ can be obtained simultaneously and thus the time taken to solve the initial-boundary-value problem (1)-(6) will be reduced significant.

\section{NUMERICAL EXPERIMENTS}

\section{A. Experiment I: Effect of Time-Step, $\ell$}

In order to test the stability and convergence properties of the novel scheme constructed in Section II, the model (1)-(6) is simulated by using $\operatorname{DM}(\theta)$ and $\operatorname{SM}(\theta)$ with the parameter values: $\gamma=0.20$ year $^{-1}, \mu=5.5 \times 10^{-5}$ day $^{-1}, r=0.0714$ day $^{-1}$, $K=1.0, \sigma=0.50$ day $^{-1}, \kappa=0.1857$ day $^{-1}, \alpha=0.0093$ day $^{-1}$ and $\beta=0.514$ day $^{-1}$. Furthermore, the following initial subpopulations are for simulation purposes: for $-2 \leq x \leq 2$,

$$
\begin{aligned}
& S(x, 0)=0.96 \exp \left(-10 x^{2}\right), E(x, 0)=0, \\
& I(x, 0)=0.04 \exp \left(-100 x^{2}\right), R(x, 0)=0,
\end{aligned}
$$

as shown in Fig. I. It shows initial proportion of susceptible and infected individuals concentrated at the origin in which the proportion of susceptible individual is greater than the 
proportion of the infected individual.

The effect of the time-step on the methods $\operatorname{SM}(\theta)$ and $\operatorname{DM}(\theta)$ with $\theta=0, \frac{1}{2}, 1$ is monitored for the different values of the diffusion rate $d_{1}$ and fixed the other diffusion rate: $d_{i}, i=2,3,4$. The numerical results are obtained using $d_{1}=0.001,0.01,0.1$ and the stability intervals of the numerical methods are shown in Table I. In the case method $\operatorname{SM}(\theta)$ with $\theta=0$ and $d_{1}=0.001$, this method produces overflow when $\ell>0.1896$ while contrived oscillations were exhibited in the numerical solutions as $\ell$ is increased beyond the value 0.1899 with $d_{1}=0.01$ and the value 0.0500 with $d_{1}=0.1$. Using $\theta=\frac{1}{2}$, method $\operatorname{SM}(\theta)$ produces overflow as $\ell>2.5533$ with $d_{1}=0.001, \ell>2.9240$ with $d_{1}=0.01$ and $\ell>2.6921$ with $d_{1}=0.1$.
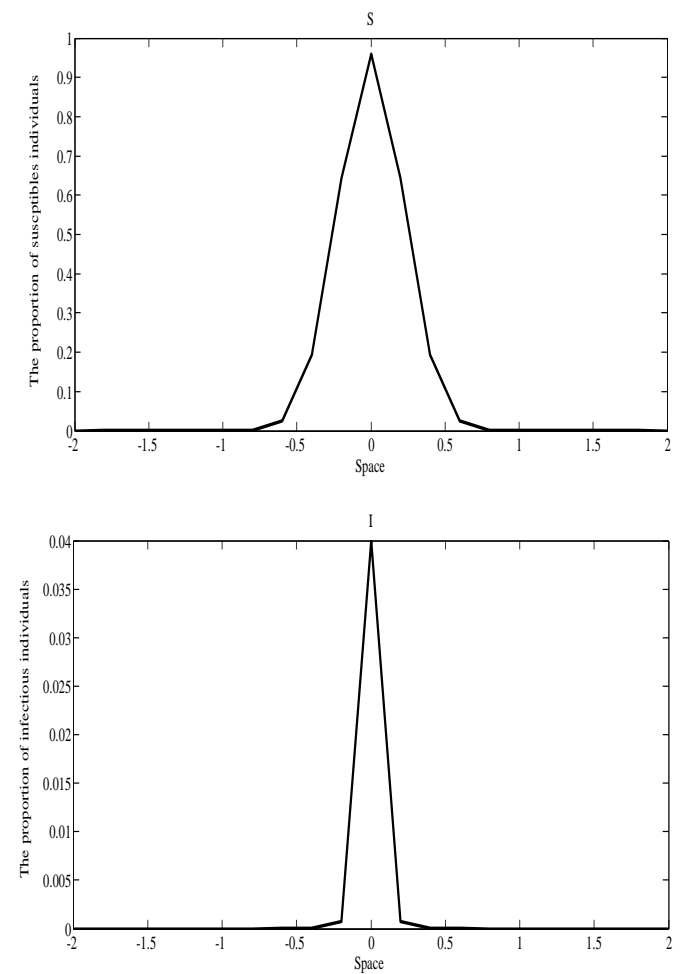

Fig. 1. The initial distributions of the proportion of susceptibles, exposed, infectious and recovered individuals for Experiments I.

\begin{tabular}{||c|c|c|c||}
\multicolumn{2}{|c|}{ TABLE I: STABILITY INTERVALS OF THE METHODS } \\
\hline \hline$d_{1}$ & $\theta$ & \multicolumn{2}{|c||}{ Interval of stability } \\
\cline { 2 - 4 } & & Method $S M(\theta)$ & Method $D M(\theta)$ \\
\hline 0.001 & 0 & $(0,0.1896)$ & $(0,0.2136)$ \\
& $\frac{1}{2}$ & $(0,2.5533)$ & $(0,11.8433)$ \\
& 1 & $(0,2.7999)$ & $(0,18.3880)$ \\
\hline 0.01 & 0 & $(0,0.1899)$ & $(0,0.2136)$ \\
& $\frac{1}{2}$ & $(0,2.9240)$ & $(0,11.0000)$ \\
& 1 & $(0,2.9243)$ & $(0,14.8312)$ \\
\hline 0.1 & 0 & $(0,0.0500)$ & $(0,0.0500)$ \\
& 1 & $(0,2.6921)$ & $(0,16.4129)$ \\
& $\frac{1}{2}$ & $(0,2.9238)$ & $(0,17.6499)$ \\
\hline
\end{tabular}

Using $\theta=1$, method $D M(\theta)$ produces overflow for $\ell>2.7999$ with $d_{1}=0.001, \ell>2.9243$ with $d_{1}=0.01$ and $\ell>2.9238$ with $d_{1}=0.1$.

For method $\operatorname{DM}(\theta)$ with $\theta=0$, this method produces overflow for $\ell>0.2136$ with $d_{1}=0.001,0.01$ and for $\ell>0.0500$ with $d_{1}=0.1$. Using $\theta=\frac{1}{2}$ and $\theta=1$ method $D M(\theta)$ produces overflow began as $\ell$ is increased above the values in the stability interval (see Table I).

As above described and Table I, it is verified that method $D M(\theta)$ has a much better stability property than method $\operatorname{SM}(\theta)$. Furthermore, the method $\operatorname{DM}(\theta)$ with $\theta=1$ will be used to simulate the model (1)-(6). The space and time steps are given the value $h=0.1$ and $\ell=0.01$, respectively. The numerical results at $t=5,10,15$ are depicted in Fig. 2. These show that the proportion of all populations spread throughout domain $[-2,2]$ as time increases. The obtained results appear to be almost coincident with Samsuzzoha et al. [1] in their Fig. 2, therefore, showing that the proposed method (14)-(17) gives a reliable representation of the numerical solutions associated with (1)-(7).
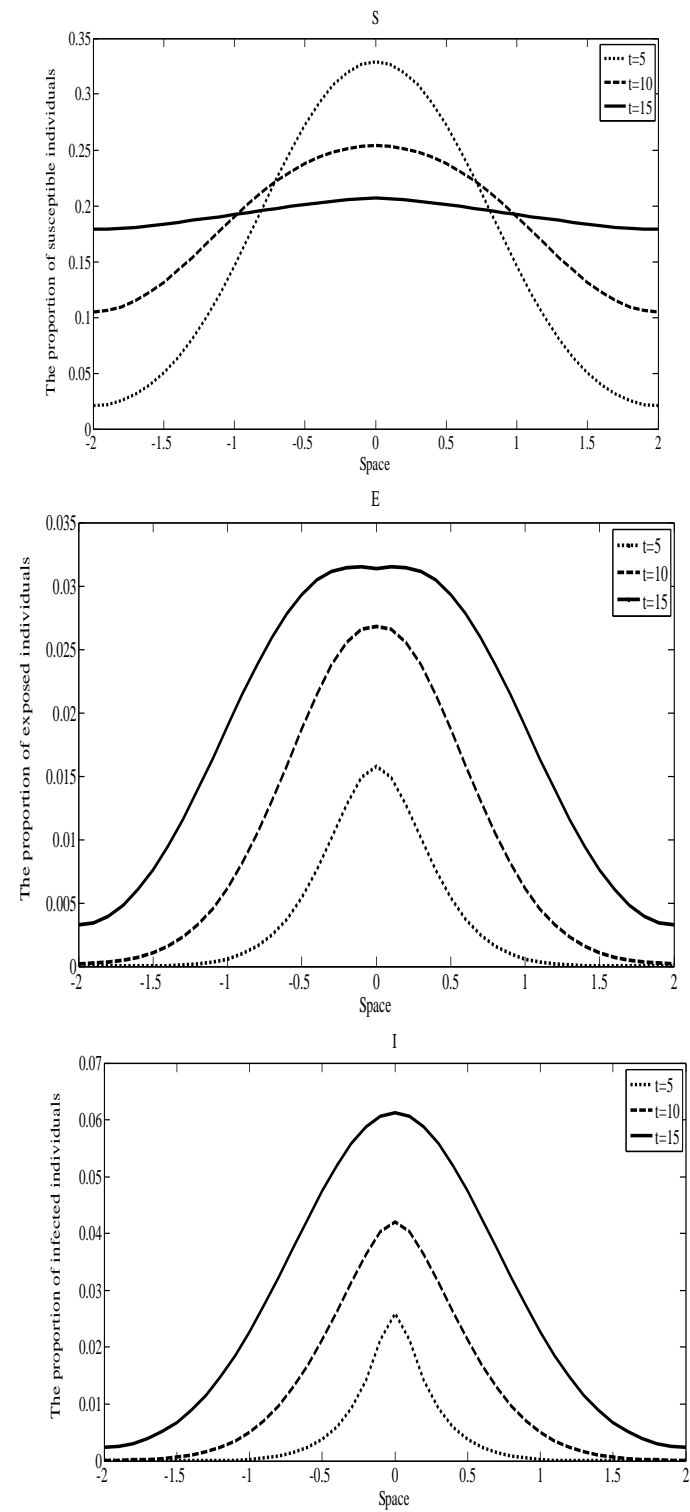


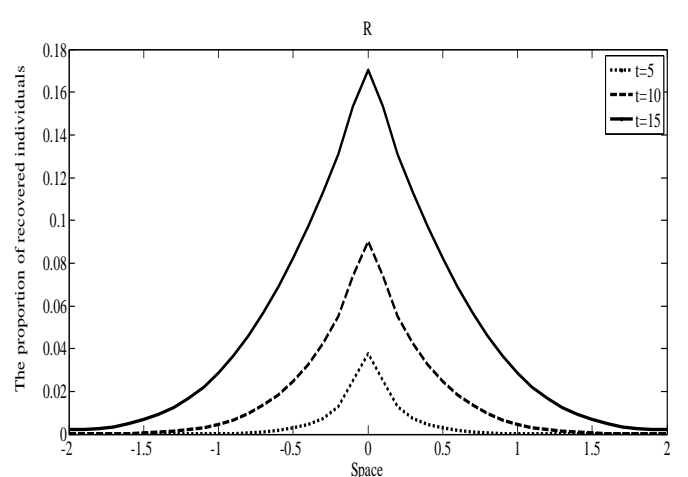

Fig. 2. Numerical simulation of the model at time $t=5,10,15$ with $h=0.1, \ell=0.01$ : a) Proportion of susceptible individual on $-2 \leq x \leq 2$; b) Proportion of exposed individual on $-2 \leq x \leq 2$; c) Proportion of infectious individual on $-2 \leq x \leq 2$; d) Proportion of recovered individual on $-2 \leq x \leq 2$

\section{CONCLUSIONS}

A first-order in time and second-order in space, finite-difference scheme has been developed and implemented in this paper for computing the solutions of the SEIR influenza model in one dimension (1)-(4). The von Neumann method is used to investigate the stability of the scheme. Numerical experiments are chosen to investigate the dynamic behavior of the model for different step-lengths. It is seen that the proposed method is more competitive (in terms of numerical stability) than the standard finite-difference method. Our results also show that the proposed method (the method $D M(\theta)$ ) produced numerically-stable solutions for the SEIR influenza model which are similar to those reported in Samsuzzoha et al. [1].

\section{REFERENCES}

[1] M. D. Samsuzzoha, M. Singh, and D. Lucy, "Numerical study of an influenza epidemic model with diffusion," Appl.Math.Comp., vol. 217 , pp. 3461-3479, 2010.
[2] E. H. Twizel, "Numerical Methods," With Applications in the Biomedical Sciences, Chichester, UK: Ellis Horwood, 1988.

[3] O. V. Baroyan, G. A. Mironov, and L. A. Rvachev, "An algorith modeling global epidemics of mutant origin," Prog. Comp. Soft., vol. 5, pp. 272-277, 1981 .

[4] O. Baroyan, V. Rvachev, and Y. Ivannikov, Modelling and Forecasting of Influenza Epidemics in the Territory of the USSR (in Russian), Moscow, 1977.

[5] V. Colizza, A. Barrat, M. Barthelemy, and A. Vespignani, "The modeling of global epidemics: Stochastic dynamics and predictabiliyt," Bull.Math. Biol., vol. 68, pp. 1893-1921, 2006.

[6] V. Colizza, A. Barrat, M. Barthelemy, and A. Vespignani, "The role of the airline transportation network in the prediction and predictability of global epidemicsy," Proc. Nat. Acad. Sci., vol. 103, pp. 2015-2020, 2006.

[7] P. Fine, "Applications of mathematical models to the epidemiology of influenza: a critique," in P. Selby (Ed.), Influenza Models, Lancaster: MTP Press Limited, 1982.

[8] L. Sattenspiel, "Modeling the Spread of Infectious Disease in Human Populations," Year. Phys. Anthro., vol. 33, pp. 245-276, 1990.

[9] Chinviriyasit, "Numerical modeling of SIR epidemic model with diffusion," Appl.Math.Comp., vol. 216, pp. 395-409, 2010.

[10] E. Massad, N. M. Bruattini, B. A. F. Coutinho, and F. L. Lopeq, "The 1918 influenza A epidemic in the city of Sao Paulo Braqil," Med. Hypoth, vol. 68, pp. 442-445, 2007.

[11] A. Klimov, L. Simonsen, K. Fukuda, and N. Cox, "Surveillance and influenza in the United States," Vacc., vol. 17, pp. 842-846, 1999.

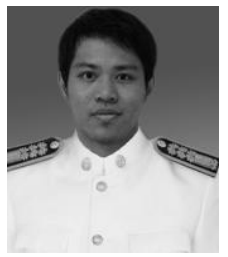

Atit Jaichuang received a B.Sc. (2005) in mathematics, and a M.Sc. (2008) in applied mathematics from Khon Kaen University. Now, he is a Ph.D. candidate, Department of Mathematics, King Mongkut's University of Technology Thonburi.

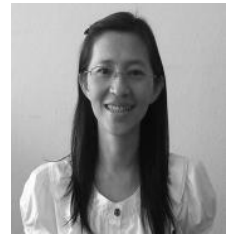

Wirawan Chinviriyasit is presently employed as associated professor doctor in mathematics at King Mongkut's University of Technology Thonburi, Thailand. She obtained her $\mathrm{PhD}$ from Brunel University (UK) in 2001. Her interested research is numerical methods of differential equations by using finite difference method, mathematical modeling in ecology and biology, applied dynamical systems, computational mathematics. 